\title{
DETERMINAÇÃO DA CURVA DE INTENSIDADE, DURAÇÃO E FREQUÊNCIA DO MUNICÍPIO DE IPAMERI - GOIÁS
}

\author{
Intensity-duration-frequency curves determination of Ipameri - Goiás
}

\author{
Daniela Carneiro Pereira ${ }^{1}$, Letícia Rodrigues Duarte ${ }^{2}$, Antover Panazzolo Sarmento ${ }^{3}$
}

Recebido em 20 de setembro de 2016; recebido para revisão em 24 de abril de 2017; aceito em 07 de agosto de 2017; disponível on-line em 17 de agosto de 2017.

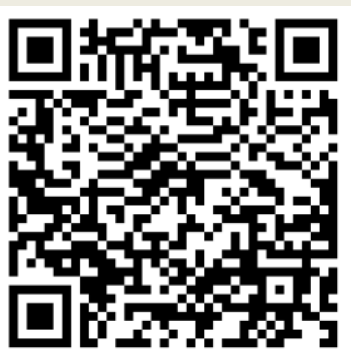

\section{PALAVRAS CHAVE:}

Intensidade-duraçãofrequência;

Gumbel;

Desagregação de chuvas; GRG - Gradiente Reduzido generalizado; Willmott.

\section{KEYWORDS:}

Intensity-dirationfrequency; Gumbel; Disaggregation of rainfall; GRG - Generalized Reduced Gradient; Willmott.

\section{* Contato com os autores:}

${ }^{1}$ e-mail: danie.pereira2010@gmail.com (D. C. Pereira )

Engenheira Civil, Universidade Federal de Goiás, Regional Catalão, Curso de Engenharia Civil.

2 e-mail: leticia.rduarte@hotmail.com ( L. R. Duarte)

Engenheira Civil, Universidade Federal de Goiás, Regional Catalão, Curso de Engenharia Civil.

3 e-mail: antoverps@gmail.com ( A.P. Sarmento)

Professor do Curso de Engenharia Civil, Universidade Federal de Goiás, Regional Catalão. engenharia é fundamental, especificadamente no estudo das chuvas intensas é feito por meio da relação entre a intensidade, a duração a frequência das mesmas. Este artigo trata da determinação de tais relações para o município de Ipameri, Goiás, devido à falta de estudos relacionados a esta localidade. Foi feito o ajustamento à distribuição estatística de Gumbel de forma a obter os valores de precipitação máxima para os períodos de retorno de 2, 5, 10, 15, 20, 25, 50 e 100 anos. Para verificação do ajuste dos dados observados a distribuição de Gumbel, realizou-se

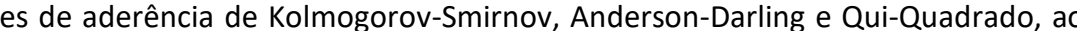

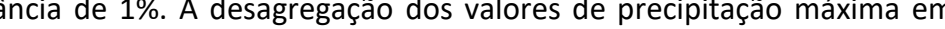
durações menores que 24 horas foi feita utilizando o método das Relações de Durações. Os parâmetros K $(881,7487)$, a $(0,1333)$, b $(10,5332)$ e c $(0,7519)$ da equação de chuvas insas foram obtidos utilizando o método do Gradiente Reduzido Generalizado. Para equação obtida foi utilizado o coeficiente de determinação $\left(R^{2}\right)$, o índice de concordância de Willmott (d) e índice de confiança (Ic), com resultados respectivos de $0,9986,0,9997$ e 0,9990. Assim esta pesquisa contribuiu para a determinação da chuvas intensas, permitindo seu uso no dimensionamento de estruturas essential, specifically with regard to the hydrological researches and structures. In this way the study of heavy rains is done relating the intensity, duration and frequency of the This paper deals with the determination of such relations for the city of Ipameri, Goiás - Brazil, due to lack of studies related to the municipality. Adjusting the statistical Gumbel distribution in order to obtain maximum precipitation values for return periods of $2,5,10,15,20,25,50$ and 100 years is made. To check the adjustment of the data observed distribution of Gumbel, we held the adherence tests (Kolmogorov-Smirnov, Anderson-Darling and Chi-Square) at a significance level of 1\%. The disaggregation of the maximum precipitation values in smaller duration than 24 hours were made using the method of durations relations. The parameters $K$ (881.7487), $a(0.1333), b(10.5332)$ and $c(0.7519)$ of equation heavy rains were obtained using the Generalized Reduced Gradient method. To validate the obtained equation was used the coefficient of determination $\left(R^{2}\right)$, the concordance index of Willmott (d) and confidence index (IC) with the results of 0.9986, 0.9997 and 0.9990. Thus, this research contributes to the determination of the intense rainfall equation, allowing its use in the design of local hydraulic structures. 


\section{INTRODUÇÃO}

As chuvas intensas, ou precipitações máximas, são aquelas responsáveis por gerar volumes de água significativos em pequenos intervalos de tempo. Devido aos grandes volumes precipitados, a ocorrência de tais eventos costuma gerar danos socioeconômicos, prejuízos ambientais, além de riscos a vida humana (FARIAS; SILVA; COELHO, 2013; QAMAR et al., 2017).

Os impactos advindos de eventos climáticos ou da ação humana no planeta têm alterado a ocorrência de chuvas intensas e, consequentemente, das inundações. Com o objetivo de diminuir os prejuízos gerados por tais inundações, as obras hidráulicas devem ser projetadas com base nos valores de vazão máxima (ARAGÃO et al., 2013; ZHAO et al., 2012; FADHEL; RICO-RAMIREZ; HAN, 2017).

O estudo e a determinação da vazão de projeto são fundamentais no dimensionamento de estruturas hidráulicas em obras de engenharia, além de ser utilizada para encontrar as cotas de alerta de inundações. Assim, sua determinação requer o uso de métodos confiáveis e seguros (STEFFEN; RONDON, 2000). A vazão de projeto pode ser estimada utilizando série de vazões históricas ou a partir de séries de precipitação, sendo a transformação feita utilizando modelos hidrológicos do tipo precipitação-vazão (TUCCl et al., 2001).

Silva e Araújo (2013) destacam a relevância dos dados de precipitação máxima para o dimensionamento de obras de engenharia, principalmente em projetos relacionados a drenagem urbana, visto que, normalmente, não se tem dados de vazão para o local em estudo.

Desse modo, o estudo e a caracterização das precipitações máximas são de suma importância na elaboração de projetos de engenharia voltados ao dimensionamento hidráulico, tais como galerias de águas pluviais, canalizações de córregos, bueiros, vertedores e canais de irrigação (MARTINEZ JÚNIOR; MAGNI, 1999).

A pesquisa das características de tais precipitações pode ser feita conhecendo-se os dados de duração, intensidade e frequência de ocorrência das mesmas. A relação entre estas variáveis é denominada, de maneira geral, de curvas de intensidade-duração-frequência (IDF), podendo ser uma ferramenta utilizada para transformações chuva-vazão (DAMÉ; TEIXEIRA; TERRA, 2008; MAMOON et al., 2014).

As relações entre as grandezas que caracterizam as precipitações máximas devem ser obtidas por meio da análise de chuvas ao longo de um período de tempo relativamente grande, de forma que seja possível estabelecer as frequências como probabilidades. Estas relações irão resultar em uma família de curvas intensidade-duração, uma para cada período de retorno (CASTRO; SILVA; SILVEIRA, 2011).

Por meio destas relações entre intensidade, duração e período de retorno, é possível obter a equação de chuvas intensas, sendo os parâmetros obtidos por meio de análise e tratamento de dados de pluviogramas, ou, na ausência destes, de pluviômetros. Neste último caso deve-se ainda utilizar a metodologia de desagregação de chuvas para os valores de precipitação máxima diária anual (BATISTA, 2013).

A falta de estações pluviográficas e de longas séries de dados de precipitação tem levado os projetistas a buscarem alternativas para o dimensionamento seguro. Dessa forma, alguns estudos foram desenvolvidos no Brasil para obtenção de valores de precipitação de menor duração partindo de registros pluviométricos diários, dado a existência de vasta rede pluviométrica no território. Tais métodos utilizam coeficientes na transformação da chuva de 24 horas em durações inferiores (OLIVEIRA et al., 2008).

Tendo em vista a carência de equações de chuvas intensas para estado de Goiás e a importância do estudo das relações de intensidade, duração e frequência, busca-se nesse trabalho determinar relações IDF para o município de Ipameri, de forma a obter maior segurança na elaboração de projetos de obras hidráulicas, tanto urbanas quanto agrícolas, do município.

Costa, Siqueira e Menezes apresentaram as equações de chuvas intensas para algumas localidades de Goiás e Sul do Tocantins, 
incluindo o município de Ipameri, sendo as estimativas feitas a partir da metodologia utilizada por Costa e Prado (2003), ou seja, por meio da espacialização das intensidades de precipitação. 0 método utilizado para tal determinação não analisou dados específicos do município em questão, sendo a estimativa elaborada com base em dados de 16 postos distribuídos na região, e os resultados adaptados para as demais cidades. Assim, o presente trabalho torna-se importante, apresentando cada etapa realizada no estudo das relações IDF do local, partindo-se de dados pluviométricos do município.

\section{OBJETIVO}

Objetivou-se com este trabalho determinar os parâmetros da equação de intensidade-duração-frequência ( $K, a, b$ e c) para o município de Ipameri, Goiás.

\section{METODOLOGIA}

\subsection{CARACTERIZAÇÃO DA ÁREA}

De acordo com o Censo Demográfico de 2010 do Instituto Brasileiro de Geografia e Estatística, o município de Ipameri possui uma população de 24.735 habitantes, com uma estimativa para 2015 de 26.373 habitantes. Possui uma unidade territorial de 4.368,991 $\left(\mathrm{km}^{2}\right)$, tendo o cerrado como bioma local, estando localizado na região Centro-Oeste do Brasil, no Estado de Goiás (IBGE, 2016).

\subsection{COLETA DOS DADOS}

A série histórica dos valores de precipitação foi obtida da Estação Meteorológica OMM: 83522, de responsabilidade do INMET (Instituto de Nacional de Meteorologia). Está localizada na latitude $-17,71^{\circ}$, longitude $-48,16^{\circ}$ e a uma altitude de 772,99 metros. A estação começou a operar no dia 10 de fevereiro do ano de 1977, estando em operação até os dias atuais. A escolha de tal estação se deve ao fato de esta ser a única com quantidade de dados suficientes, permitindo a realização do estudo e a obtenção de resultados confiáveis.

Os dados disponíveis de precipitação são valores diários e são disponibilizados através do Banco de Dados Meteorológicos para Ensino e Pesquisa (BDMEP). Esse banco de dados contém, na forma digital, séries históricas de estações meteorológicas convencionais da rede de estações do INMET, com diversas informações de medições diárias.

\subsection{ANÁLISE DOS DADOS}

Foram analisados valores de precipitação diária dos anos de 1977 a 2016, separando-se manualmente o acumulado de precipitação de cada mês para todos os anos, destacando-se os meses que não possuíam todos os valores de precipitação diária. Foi possível obter a precipitação média mensal utilizando todos os anos de dados acessíveis, e partindo desses valores foi determinado o ano hidrológico, correspondente a um intervalo fixo de 12 meses, começando no início do período chuvoso e terminando no fim da estação seca, segundo Naghettini e Pinto (2007).

Na Figura 1 apresentam-se as médias de precipitação de todos os anos da série histórica disponível para o município de Ipameri. É possível observar que julho se destaca como o mês de menor média de precipitação. Identifica-se também que os 6 meses mais chuvosos são outubro, novembro, dezembro, janeiro, fevereiro e março, constituindose assim o período chuvoso. Abril, maio, junho, julho, agosto e setembro constituem o período de seca, o ano hidrológico então começa em outubro e vai até setembro.

Determinado o ano hidrológico foram selecionados, também de forma manual, os anos hidrológicos completos, ou seja, aqueles em que se tem todos os valores de precipitação diária, estes foram utilizados na determinação dos valores de máxima precipitação anual. O tamanho da série histórica encontrada foi de 25 anos hidrológicos completos, variando entre 1982 e 2015.

Para cada ano hidrológico completo determinou-se a precipitação máxima diária anual, sendo esta correspondente ao maior valor de precipitação ocorrido em um dia ao longo do ano em questão. 


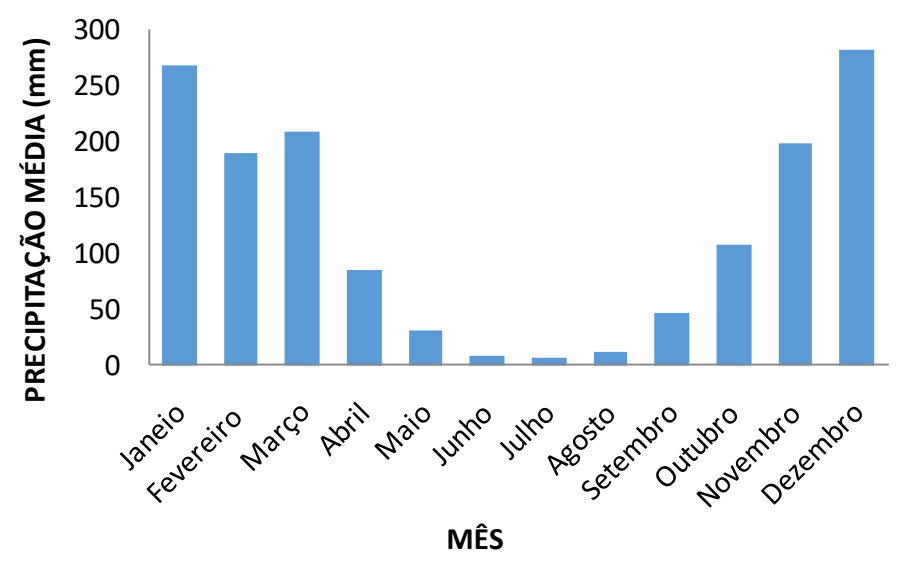

FIGURA 1: Média dos valores de precipitação mensal disponíveis para o município de Ipameri - GO, no período de 1977 a 2016.

FONTE: Autoria própria.

\subsection{AJUSTE A DISTRIBUIÇÃO ESTATÍSTICA}

Para ajuste dos valores de máxima precipitação diária anual encontrados, foi utilizada a distribuição estatística de Gumbel. Essa escolha se justifica por ser a distribuição de extremos mais utilizada na análise de frequência de variáveis hidrológicas, além de apresentar vantagens em relação as demais, pois para o seu uso não é preciso consultar tabelas de probabilidade, de forma que o ajuste é feito apenas utilizando a média e o desvio padrão dos valores de precipitação máxima diária anual. Além de tais fatores, diversos autores (RODRIGUES et al., 2008; MORUZZI; OLIVEIRA, 2009; GARCIA et al., 2011; DE PAOLA et al., 2014; ABUBAKARI; KUSI; XIAOHUA, 2017; SHRESTHA et al., 2017; VU; RAGHAVAN; LIONG, 2017) utilizaram a distribuição de Gumbel na determinação das relações de intensidade, duração e frequência de chuvas, no Brasil e em diversos países.

O ajuste foi feito utilizando a Equação 1 (CHOI; $\mathrm{CHOI}, 1999)$, a qual emprega os parâmetros estatísticos de média e desvio padrão dos valores de máxima precipitação anual para determinação da altura máxima de precipitação de 1 dia, correspondente a diferentes tempos de recorrência.

$\mathrm{x}=\overline{\mathrm{x}}-\mathrm{s}\left\{0,45+0,7797 \ln \left[\ln \frac{\operatorname{Tr}}{\operatorname{Tr}-1}\right]\right\}$
Em que:

$\mathbf{x}=$ precipitação máxima ajustada $(\mathrm{mm})$;

$\overline{\mathbf{x}}=$ média dos valores de precipitação máxima coletados $(\mathrm{mm})$;

$\mathbf{s}=$ desvio padrão das precipitações máximas anuais $(\mathrm{mm})$;

$\mathrm{Tr}=$ tempo de recorrência (anos).

A média e o desvio padrão foram calculados aplicando as Equações 2 e 3 (LANNA, 2001).

$$
\overline{\mathrm{x}}=\frac{\sum_{\mathrm{i}=1}^{\mathrm{n}} \mathrm{xi}}{\mathrm{n}}
$$

Em que:

$\overline{\mathbf{x}}=$ média aritmética $(\mathrm{mm})$;

$\mathbf{x}_{\mathbf{i}}=$ realizações da variável $(\mathrm{mm})$;

$\mathbf{n}=$ número total de dados.

$$
s=\sqrt{\frac{\sum_{\mathrm{i}=1}^{\mathrm{n}}(\mathrm{xi}-\overline{\mathrm{x}})^{2}}{\mathrm{n}-1}}
$$

Em que:

$\overline{\mathbf{x}}=$ média aritmética $(\mathrm{mm})$;

$\mathbf{x}_{\mathbf{i}}=$ realizações da variável $(\mathrm{mm})$;

$\mathbf{s}=$ desvio padrão $(\mathrm{mm})$;

$\mathbf{n}=$ número total de dados.

Foram escolhidos para este estudo os tempos de recorrência de 2, 5, 10, 15, 20, 25, 50 e 100 anos, por serem valores frequentemente utilizados para determinação de chuvas intensas. 


\subsection{TESTE DE ADERÊNCIA}

Para avaliar a qualidade do ajuste dos dados à distribuição estatística, foram realizados três diferentes testes de aderência, sendo eles: Kolmogorov-Smirnov, Anderson-Darling e Qui-Quadrado.

Os testes foram feitos com o objetivo de verificar se a distribuição de Gumbel se ajusta de forma correta e precisa aos valores de precipitação máxima anual. Para realização dos testes foi considerado um nível de significância de $1 \%$ $(\alpha=0,01)$, sendo este valor utilizado em estudos para determinação de equações de chuvas intensas, tais como os de Oliveira et al. (2005), Sharma e Singh (2010) e Oliveira et al. (2011).

Para cada precipitação máxima anual foi calculada a distribuição empírica de probabilidade (frequência), utilizando o método Califórnia (Equação 4).

$$
\mathrm{F}=\frac{\mathrm{m}}{\mathrm{n}}
$$

Em que:

$\mathbf{F}=$ frequência de ocorrência (adimensional);

$\mathbf{m}=$ ordem de determinado evento;

$\mathbf{n}=$ número de anos de observação.

Os valores da distribuição de Gumbel correspondente a cada valor de chuva máxima foi obtido pela sua função de probabilidade (Equação 5) (SUBRAMANYA, 2013; GHOSH; MISTRI, 2013).

$$
\mathrm{P}=1-\mathrm{e}^{-\mathrm{e}^{-\mathrm{y}}}
$$

Em que:

$\mathbf{P}=$ probabilidade (adimensional);

$\mathbf{e}=$ base dos logaritmos neperianos (adimensional);

$\mathbf{y}=$ variável reduzida de Gumbel (adimensional).

Partindo dos valores de precipitação da distribuição empírica e da distribuição teórica
(Gumbel), calculou-se as estatísticas para os testes de aderência.

\subsection{DESAGREGAÇÃO DA CHUVA}

A chuva máxima anual, referente a 1 dia de precipitação, foi desagregada em durações menores, sendo esse procedimento necessário quando não se tem dados de pluviógrafos. 0 método utilizado foi o de relações de durações (CETESB, 1986). A escolha do método se justifica por ser de uso simples, oferecendo resultados satisfatórios na determinação de alturas de precipitação com duração inferior a 1 dia.

Os valores de precipitação máxima anual para cada tempo de recorrência foram desagregados em durações menores que $1 \mathrm{dia}$. As durações utilizadas foram $5,10,15,20,25,30,60$, 120, 180, 360, 480, 600, 720 e 1440 minutos. Para obtenção da precipitação nas durações citadas, procedeu-se multiplicando a precipitação de duração maior pelo coeficiente que a transforma na precipitação de menor duração, os coeficientes e suas transformações correspondentes são mostrados no Quadro 1.

Primeiramente é preciso obter a precipitação de 24 horas, sendo esta diferente da chuva de 1 dia. Segundo Bertoni e Tucci (2001), a chuva de 24 horas é o total máximo precipitado, equivalente a um período contínuo de 24 horas, e não corresponde necessariamente ao período de observação. Já a precipitação de 1 dia é o valor contido entre os horários de observação pluviométrica.

Assim sendo, é necessário multiplicar o valor da precipitação diária pelo coeficiente 1,14 (Quadro 1). Essa multiplicação permite encontrar o valor da chuva de 24 horas. A partir da precipitação de 24 horas (1.440 minutos) determina-se a precipitação de 12 horas (720 minutos) multiplicando-se pelo coeficiente 0,85 , também mostrado no Quadro 1. O mesmo raciocínio é feito para as demais durações, multiplicando sempre o coeficiente de desagregação pela precipitação de 24 horas. 


\section{QUADRO 1: Coeficientes de desagregação de chuva de 24 horas utilizando o método das Relações de Durações.}

\begin{tabular}{|c|c|}
\hline Relação entre Alturas Pluviométricas & Coeficiente de Desagregação \\
\hline 5 min para 30 min & 0,34 \\
\hline 10 min para 30 min & 0,54 \\
\hline 15 min para 30 min & 0,70 \\
\hline 20 min para 30 min & 0,81 \\
\hline 25 min para 30 min & 0,91 \\
\hline 30 min para 1 h & 0,74 \\
\hline $1 \mathrm{~h}$ para 24h & 0,42 \\
\hline $2 \mathrm{~h}$ para $24 \mathrm{~h}$ & 0,48 \\
\hline $3 \mathrm{~h}$ para $24 \mathrm{~h}$ & 0,54 \\
\hline $6 \mathrm{~h}$ para $24 \mathrm{~h}$ & 0,72 \\
\hline $8 \mathrm{~h}$ para $24 \mathrm{~h}$ & 0,78 \\
\hline $10 \mathrm{~h}$ para $24 \mathrm{~h}$ & 0,82 \\
\hline $12 \mathrm{~h}$ para $24 \mathrm{~h}$ & 0,85 \\
\hline $24 \mathrm{~h}$ para $1 \mathrm{dia}$ & 1,14 \\
\hline
\end{tabular}

FONTE: CETESB (1986) apud ARAGÃO et al. (2013).

\begin{tabular}{|c|c|}
\hline Duração (minutos) & Precipitação Adotada (mm) \\
\hline 5 & 8 \\
\hline 10 & 10 \\
\hline 15 & 15 \\
\hline 20 & 15 \\
\hline 30 & 20 \\
\hline 45 & 23 \\
\hline 60 & 25 \\
\hline 120 & 30 \\
\hline 180 & 33 \\
\hline 240 & 35 \\
\hline 360 & 40 \\
\hline 480 & 40 \\
\hline 720 & 47 \\
\hline 1440 & 55 \\
\hline
\end{tabular}

FONTE: CETESB (1986).

Para o caso da duração de 30 minutos o valor de referência a ser multiplicado pelo coeficiente é a precipitação de 1 hora. Para as durações menores que 30 minutos, o respectivo coeficiente de desagregação deve ser multiplicado pela chuva de 30 minutos. 0 procedimento deve ser feito para cada tempo de recorrência separadamente, obtendo para cada um deles a lâmina de precipitação para diferentes durações.

Valores mínimos de precipitação devem ser obedecidos, estes estão apresentados no
Quadro 2, onde para cada duração, tem-se um valor mínimo de precipitação correspondente. Se ao desagregar a chuva forem obtidos valores inferiores aos mínimos tabelados, deve-se desconsiderá-los e adotar os mínimos.

Tendo-se os valores de precipitação, as respectivas intensidades são obtidas dividindo-se tais valores pela duração correspondente. Encontrando-se as intensidades é possível gerar as curvas IDF para os dados analisados, sendo uma para cada tempo de recorrência. 


\subsection{DETERMINAÇÃO DOS PARÂMETROS DA EQUAÇÃO IDF}

Para determinar os parâmetros $(K, a, b$ e $c)$ da equação de chuvas intensas para o município em questão foi utilizado o Solver, ferramenta computacional disponível no programa Excel ${ }^{\circledR}$. Uma das formas utilizadas pelo Solver na resolução de problemas é o Método do Gradiente Reduzido Generalizado (GRG), utilizado na otimização e solução de problemas não lineares.

A determinação dos parâmetros é feita com base nos valores de precipitação observados e estimados. A intensidade observada se refere aos valores de intensidade já obtidos por Gumbel e Desagregação. A intensidade estimada é obtida aplicando-se a Equação 6 (SOUZA et al., 2012; CARDOSO et al. 2014, CAMPOS et al., 2017).

$$
\mathrm{i}=\frac{\mathrm{KTr}^{a}}{(\mathrm{t}+\mathrm{b})^{\mathrm{c}}}
$$

\section{Em que:}

$\mathbf{i}=$ intensidade de precipitação $\left(\mathrm{mm} \mathrm{h}^{-1}\right)$;

$\mathrm{Tr}=$ tempo de recorrência (anos);

$\mathbf{t}=$ duração da chuva (minutos);

$\mathbf{K}$, b e $\mathbf{c}=$ parâmetros que descrevem características locais (adimensional);

$\mathbf{a}=$ parâmetro regional constante (adimensional).

Para a aplicação do Solver na determinação das estimativas de precipitação e dos parâmetros da equação de chuvas intensas, fez-se necessário calcular a soma quadrática total (SQT), estabelecida como o resultado a adição entre a soma dos quadrados dos resíduos (SQRes) e a soma dos quadrados devido a regressão (SQReg).

A determinação da soma dos quadrados total (SQT) foi feita partindo-se da diferença entre os valores observados e a média dos mesmos. Tal subtração deve ser elevada ao quadrado e somados todos os termos. A soma dos quadrados devidos à regressão (SQReg) foi feita da mesma forma, porém foram utilizados os valores estimados, ao invés dos valores observados. A SQRes é obtida através dos valores observados menos os valores estimados, sendo os valores elevados ao quadrado e posteriormente somados.
Determinados os valores da SQT, SQReg e da SQRes, aplicou-se o GRG através do Solver, de maneira que o objetivo $S Q T=S Q R e g+S Q R e s$, ou seja, SQT - (SQReg + SQRes) fosse nulo.

Foi preciso informar também para a resolução no Solver quais devem ser as células variáveis, ou seja, quais os parâmetros que podem ser alterados para alcançar o objetivo proposto. Neste caso os parâmetros variáveis e que se desejava determinar eram $K, a, b$ e $c$ da equação de chuvas intensas.

Como se espera um valor nulo (ou próximo de zero) para SQRes, adicionou-se essa restrição nos parâmetros do Solver, pois quanto mais próximo de zero for o valor do SQRes, melhor a qualidade do ajuste.

De forma resumida a determinação dos parâmetros IDF foi feita, definindo-se: (i) o objetivo $(S Q T-(S Q R e g+S Q R e s)=0)$, (ii) as células variáveis $(K, a, b$ e c), (iii) a restrição (SQRes $=0)$ e (iv) o Método GRG. Feito isto, encontrou-se os valores dos parâmetros IDF que mais se adequam ao objetivo proposto.

\subsection{AVALIAÇÃO DA EQUAÇÃO}

Para avaliar os coeficientes estimados pela metodologia proposta para a equação IDF encontrada foram utilizados três parâmetros, o coeficiente de determinação $\left(R^{2}\right)$, o índice de concordância de Willmott $(d)$ e o coeficiente de confiança (Ic). O objetivo destes testes é avaliar o quão próximos estão os valores estimados pela equação determinada dos valores observados.

$O R^{2}$ é obtido pela divisão da SQReg pela $S Q T$, devendo sempre ser um valor positivo, variando de 0 a 1 . Este coeficiente é utilizado na avaliação do ajuste dos parâmetros da equação de chuvas intensas.

O índice de concordância de Wilmott $(d)$ determina a exatidão do método utilizado, e avalia o grau de afastamento entre os valores estimados e observados, variando também entre 0 e 1 . O seu cálculo é efetuado de acordo com a Equação 7 (WILLMOTT, 1981). 


$$
\mathrm{d}=1-\left[\frac{\sum(\mathrm{Pi}-\mathrm{Oi})^{2}}{\sum(|\mathrm{Pi}-\mathrm{O}|+|\mathrm{Oi}-\mathrm{O}|)^{2}}\right]
$$

Em que:

$$
\begin{aligned}
& \mathbf{P i}=\text { valor estimado }\left(\mathrm{mm} \mathrm{h}^{-1}\right) ; \\
& \left.\mathbf{O i}=\text { valor observado }\left(\mathrm{mm} \mathrm{h}^{-1}\right)\right) ; \\
& \mathbf{O}=\text { média dos valores observados }\left(\mathrm{mm} \mathrm{h}^{-1}\right) .
\end{aligned}
$$

A partir da Equação 8 foi calculado o coeficiente de confiança (Ic) proposto por Camargo e Sentelhas (1997).

Para tal fim, calculou-se também o coeficiente de correlação ( $r$ ), de acordo com a Equação 9.

$$
\text { Ic }=\mathrm{r} \times \mathrm{d}
$$

\section{Em que:}

Ic = coeficiente de confiança (adimensional); $\mathbf{r}=$ coeficiente de correlação (adimensional); d = índice de concordância de Willmott (adimensional).

$$
r=\frac{\sum(x-\bar{x})(y-\bar{y})}{\sqrt{\sum(x-\bar{x})^{2}(y-\bar{y})^{2}}}
$$

\section{Em que:}

$r=$ coeficiente de correlação (adimensional);

$\mathbf{x}=$ valores observados $\left(\mathrm{mm} \mathrm{h}^{-1}\right)$;

$\overline{\mathbf{x}}=$ média dos valores observados $\left(\mathrm{mm} \mathrm{h}^{-1}\right)$;

$\mathbf{y}=$ valores estimados $\left(\mathrm{mm} \mathrm{h}^{-1}\right)$;

$\overline{\mathbf{y}}=$ média dos valores estimados $\left(\mathrm{mm} \mathrm{h}^{-1}\right)$.

\section{RESULTADOS E DISCUSSÃO}

\subsection{DADOS DE CHUVA}

Foram selecionados manualmente os anos hidrológicos completos, obtendo um total de 25 anos. Foram também identificados os valores de precipitação máxima anual correspondente a cada um deles. Na Tabela 1 apresenta-se os valores de precipitação obtidos com base na série histórica de precipitação do BDMEP da estação 83522, instalada no município de Ipameri.

Ao analisar as precipitações máximas de 1 dia (Tabela 1), percebe-se que o ano hidrológico que possui maior lâmina de precipitação, entre os anos analisados, é o de 1997/1998, ou seja, abrange o período entre setembro de 1997 e agosto de 1998.

Nota-se a oscilação dos eventos máximos, com destaque dado aos anos 1983/1984, 1988/1989, 1997/1998, 2009/2010 e 2011/2012, que possuem altura de precipitação máxima diária superior a $100 \mathrm{~mm}$. Com relação ao menor valor máximo, destaca-se o ano 1984/1985, com menos de $60 \mathrm{~mm}$ de precipitação máxima.

\subsection{TRATAMENTO DOS DADOS}

A estimativa dos valores de precipitação, para cada tempo de recorrência selecionado, foi realizada a partir da distribuição estatística de Gumbel. Para tal, foi necessário determinar os valores de média e desvio padrão referentes as máximas precipitações anuais presentes na Tabela 1, conforme apresentado na Tabela 2. Aplicando a Equação 3 foi possível obter a altura máxima de precipitação de 1 dia para os tempos de recorrência de $2,5,10,15,20,25,50$ e 100 anos (Tabela 3). 
TABELA 1: Anos hidrológicos completos e suas respectivas precipitações máximas diárias anuais para o município de Ipameri.

\begin{tabular}{cc|cc}
$\begin{array}{c}\text { Ano } \\
\text { hidrológico }\end{array}$ & $\begin{array}{c}\text { Precipitação máxima } \\
\text { diária anual }(\mathbf{m m})\end{array}$ & $\begin{array}{c}\text { Ano } \\
\text { hidrológico }\end{array}$ & $\begin{array}{c}\text { Precipitação máxima } \\
\text { diária anual (mm) }\end{array}$ \\
\hline- & - & $2002 / 2003$ & 67,3 \\
\hline $1982 / 1983$ & 71,5 & $2003 / 2004$ & 63,0 \\
\hline $1983 / 1984$ & 109,0 & $2004 / 2005$ & 98,3 \\
\hline $1984 / 1985$ & 58,4 & $2005 / 2006$ & 99,1 \\
\hline $1988 / 1989$ & 107,2 & $2006 / 2007$ & 77,8 \\
\hline $1990 / 1991$ & 59,2 & $2007 / 2008$ & 64,0 \\
\hline $1992 / 1993$ & 72,8 & $2008 / 2009$ & 64,8 \\
\hline $1996 / 1997$ & 76,2 & $2009 / 2010$ & 106,3 \\
\hline $1997 / 1998$ & 119,1 & $2010 / 2011$ & 96,4 \\
\hline $1998 / 1999$ & 65,0 & $2011 / 2012$ & 106,2 \\
\hline $1999 / 2000$ & 88,2 & $2012 / 2013$ & 68,6 \\
\hline $2000 / 2001$ & 96,6 & $2013 / 2014$ & 85,8 \\
\hline $2001 / 2002$ & 84,4 & $2014 / 2015$ & 93,6 \\
\hline & & & FONTE: Autoria Própria. \\
\hline
\end{tabular}

TABELA 2: Média e desvio padrão dos valores de precipitação máxima anual dos anos hidrológicos em análise.

\begin{tabular}{cc} 
Média $(\mathrm{mm})$ & Desvio padrão $(\mathbf{m m})$ \\
\hline 83,9520 & 18,2364 \\
\hline & FONTE: Autoria Própria.
\end{tabular}

TABELA 3: Estimativa da precipitação máxima pela distribuição estatística de Gumbel, realizada para cada tempo de recorrência.

\begin{tabular}{cc} 
Tr (anos) & Precipitação máxima de 1 dia (mm) \\
\hline 2 & 80,96 \\
\hline 5 & 97,07 \\
\hline 10 & 107,74 \\
\hline 15 & 113,76 \\
\hline 20 & 117,98 \\
\hline 25 & 121,23 \\
\hline 50 & 131,23 \\
\hline 100 & 141,15 \\
\hline
\end{tabular}

FONTE: Autoria Própria.

A análise da Tabela 3 permite verificar, conforme o esperado, que quanto maior o tempo de recorrência, maior é a lâmina prevista de precipitação, ou seja, a medida que o tempo de recorrência aumenta, maiores são os valores de precipitação que podem vir a ocorrer neste período de referência.

\subsection{TESTE DE ADERÊNCIA}

$\mathrm{Na}$ Figura 2 apresenta-se 0 comportamento da série de precipitações máximas anuais, com a frequência de precipitação observada encontrada pelo método empírico de Califórnia, e a curva de distribuição estimada pelo modelo de Gumbel, relativo ao município de Ipameri. É possível 
perceber uma boa aderência entre a distribuição de valores observados e os estimados pela distribuição teórica.

Para o teste de Kolmogorov-Smirnov, o valor de $D$ observado foi de 0,1521. Para um tamanho de amostra igual a $25(\mathrm{~N}=25)$, obteve-se o valor de $D_{\mathrm{N}, \alpha}$ de 0,317 (NAGHETTINI; PINTO, 2007), considerando um nível de significância de 0,01 . Comparando os dois resultados, percebe-se que $D<D_{N, \alpha}$, portanto a hipótese é aceita, ou seja, a distribuição de Gumbel pode ser utilizada para representar o comportamento dos valores de precipitação máxima analisados.

No teste de Anderson-Darling o valor encontrado para a estatística foi de 0,8237. Como a distribuição utilizada foi Gumbel, deve-se utilizar o fator de correção $(1+0,2 / \sqrt{N})$ (NAGHETTINI; PINTO, 2007). Portanto, a estatística $W 2 n$ foi de 0,8567 . Para um nível de significância de 0,01 , temse o valor de 1,038 para $W^{2}{ }_{\text {crit }, \alpha}$. Comparando os dois resultados é possível perceber que $\mathrm{W}^{2}{ }_{\mathrm{n}}<\mathrm{W}^{2}{ }_{\text {crit, } \alpha} \mathrm{e}$, portanto, o ajuste a distribuição probabilística de Gumbel é aceitável.

Para o teste do Qui-Quadrado (WATANABE, 2013) o número de intervalos de cada classe foi de 5.0 valor encontrado para a estatística foi de 0,4276 , com 2 graus de liberdade. Para o nível de significância de $1 \%$ o valor crítico é de 9,2103, portanto, a distribuição teórica pode representar os valores observados.

\subsection{DESAGREGAÇÃO DA CHUVA DIÁRIA}

Partindo dos valores de precipitação máxima diária (Tabela 3), foi aplicado o Método das Relações de Durações. Dessa forma, obteve-se os valores de precipitação para durações inferiores a 1 dia, correspondentes a cada tempo de recorrência utilizado. A intensidade de precipitação máxima foi determinada através da divisão dos valores de precipitação por suas respectivas durações.

Ocorre variação diretamente proporcional entre a precipitação e a duração, ou seja, quanto maior a duração da chuva maior o volume precipitado. $\mathrm{O}$ mesmo acontece ao se analisar o tempo de recorrência, de maneira que a precipitação aumenta com o aumento deste.

O contrário ocorre ao analisar a intensidade de precipitação com a duração, variando de maneira inversamente proporcional, para um mesmo tempo de recorrência, sendo esse comportamento típico ao se relacionar intensidade e duração das chuvas, segundo Bertoni e Tucci (2001).

Na Figura 3 apresenta-se as curvas IDF de Ipameri, evidenciando o comportamento típico das curvas.

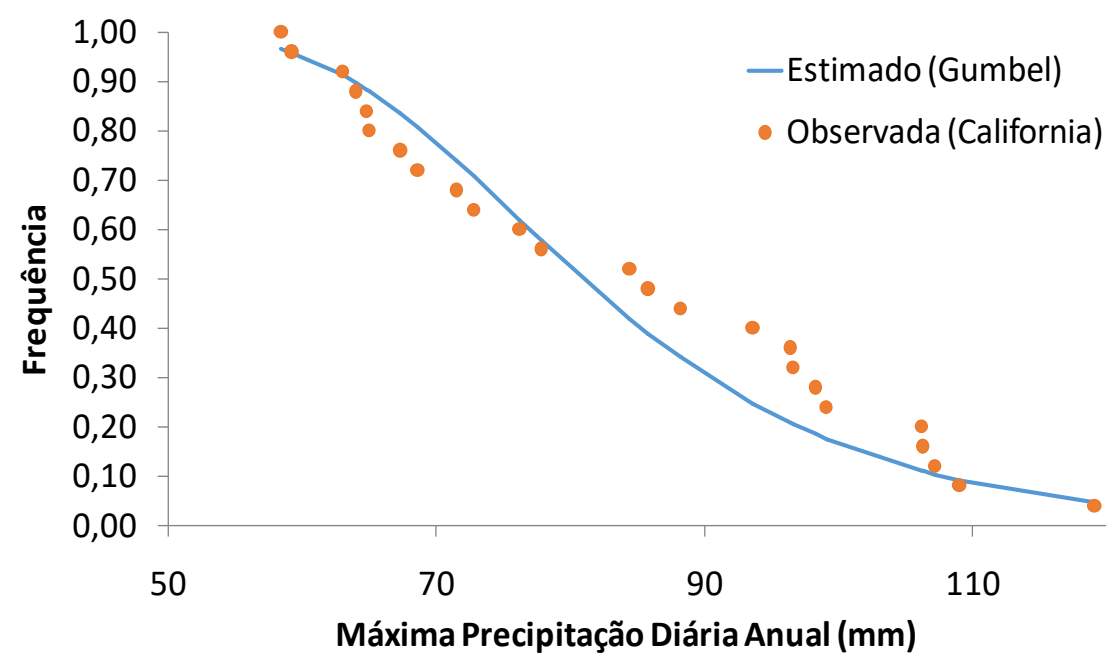

FIGURA 2: Distribuição dos valores máximos de precipitação anual observados e estimados pelo modelo de Gumbel, para o município de Ipameri - GO.

FONTE: Própria autoria. 


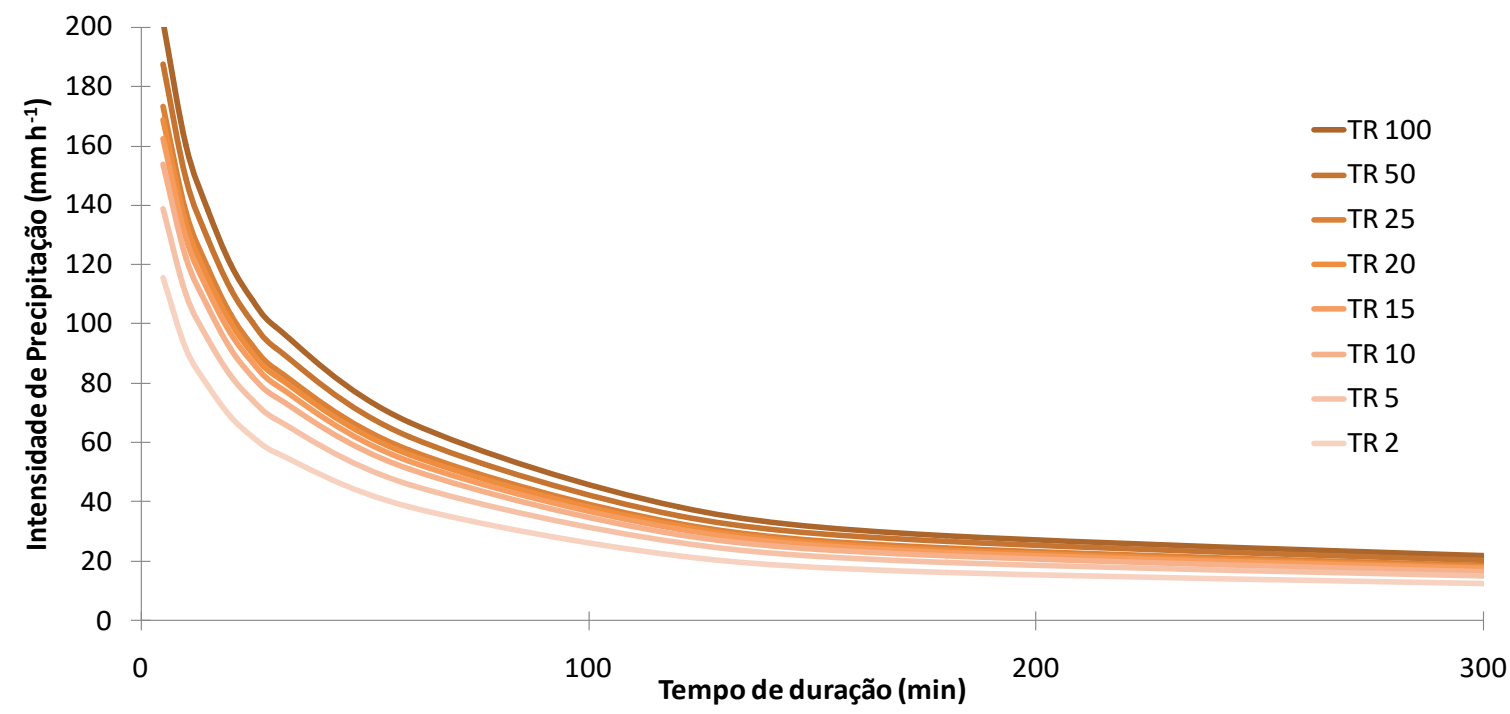

FIGURA 3: Valores de intensidade de precipitação em função do tempo para diferentes tempos de recorrência para o município de Ipameri - GO. FONTE: Autoria própria.

\subsection{DETERMINAÇÃO DOS PARÂMETROS IDF}

A determinação dos parâmetros $K, a, b$ e $c$ da equação de chuvas intensas de Ipameri foi feita ajustando-se a equação geral aos dados pluviométricos do município, por meio do Método do Gradiente Reduzido Generalizado. Substituindo os parâmetros estimados na equação de chuvas intensas (Equação 6), admitindo-se quatro casas decimais, obtém-se a equação IDF específica para o município de Ipameri (Equação 10).

$$
\mathrm{i}=\frac{881,7487 \cdot \operatorname{Tr}^{0,1333}}{(\mathrm{t}+10,5332)^{0,7519}}
$$

A equação foi obtida tendo-se como base a utilização de diferentes durações, que variam de 5 a 1440 minutos, além de serem empregados valores significativos de tempo de recorrência $(2,5,10,15$, $20,25,50$ e 100 anos). Dado este fato, a equação determinada possui uma grande faixa de aplicação, podendo ser empregada na estimativa da precipitação máxima utilizada para elaboração de diversos projetos hidráulicos a serem implantados no município de Ipameri.

\subsection{AVALIAÇÃO DA EQUAÇÃO IDF}

Foram utilizados três indicadores para avaliação da equação de chuvas intensas proposta neste trabalho. Um deles foi o coeficiente de determinação, que apresentou boa qualidade no ajuste da equação, com valor de $R^{2}$ igual a 0,9986. Na Figura 4 apresenta-se a relação entre os valores estimados e observados, bem como a linha de tendência dos valores e o $\mathrm{R}^{2}$.

$O$ índice de concordância de Willmott também foi utilizado na avaliação da equação obtida e apresentou um bom desempenho, com valor de 0,9997.

Complementarmente calculou-se o coeficiente de confiança (Ic) proposto por Camargo e Sentelhas (1997), que teve como resultado o valor de 0,9990, obtido através do produto do índice de Willmott $(0,9997)$ e do coeficiente de correlação $(0,9993)$, sendo o desempenho considerado como ótimo.

Na Tabela 4 apresentam-se os desvios relativos médios entre os valores observados $\mathrm{e}$ estimados pela equação proposta relativos a cada tempo de recorrência utilizado.

O maior desvio médio (variação do valor estimado pelo observado dividida pelo valor observado) obtido para o tempo de recorrência de 2 anos, superestimando a precipitação em 5,42\%, sendo este valor relativamente baixo, e garantindo a confiabilidade dos parâmetros estimados para a equação de chuvas intensas do município de Ipameri. Os desvios médios considerando cada duração foram analisados, sendo os valores máximos positivos e negativos, respectivamente, de 6,59 e -3,57 (Tabela 4). 


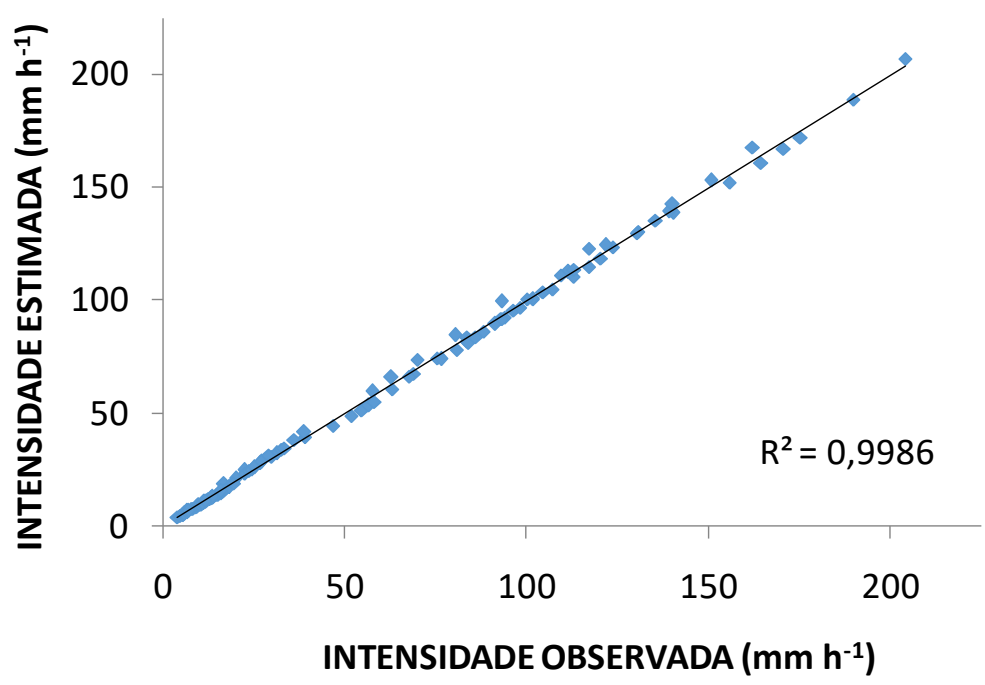

Figura 4: Relação entre intensidade máxima de precipitação observada e estimada, linha de tendência e o valor de $\mathrm{R}^{2}$, correspondentes aos dados do município de Ipameri - GO.

FONTE: Autoria própria.

\begin{tabular}{|c|c|c|c|}
\hline $\operatorname{Tr}$ (anos) & Desvio médio (\%) & $\mathrm{t}$ (minutos) & Desvio médio (\%) \\
\hline 2 & 5,42 & 5 & $-0,36$ \\
\hline 5 & $-0,65$ & 10 & 1,72 \\
\hline 10 & $-1,82$ & 15 & $-0,08$ \\
\hline 15 & $-1,85$ & 20 & 0,64 \\
\hline 20 & $-1,66$ & 25 & $-0,09$ \\
\hline 25 & $-1,40$ & 30 & $-1,18$ \\
\hline 50 & $-0,10$ & 60 & $-3,57$ \\
\hline 100 & 1,87 & 120 & 6,23 \\
\hline- & - & 180 & 6,59 \\
\hline- & - & 360 & $-3,04$ \\
\hline- & - & 480 & $-3,36$ \\
\hline- & - & 600 & $-2,53$ \\
\hline- & - & 720 & $-1,40$ \\
\hline- & - & 1440 & 0,08 \\
\hline
\end{tabular}

Os desvios entre os valores observados e estimados podem ser considerados muito pequenos, quando comparados aos desvios relativos médios obtidos por Oliveira et al. (2005), no estudo de chuvas intensas para localidades de Goiás e Distrito Federal. De maneira geral, os desvios apresentados pelos autores citados variaram de $-1,6$ a 43,9\%, para alguns municípios da região.

\section{CONCLUSÃO}

Utilizou-se o método de Relações entre Durações para desagregação dos valores de precipitação e, pelo método do GRG, obteve-se os parâmetros da equação de chuvas intensas para o município de Ipameri - GO, tendo como resultado $K=881,7487, a=0,1333, b=10,5332, c=0,7519$. A equação IDF determinada neste trabalho poderá 
contribuir para o dimensionamento de projetos hidrológicos no município de Ipameri - GO.

A metodologia aplicada neste estudo para determinação das relações de intensidade, duração e frequência das chuvas intensas para o município de Ipameri obteve resultados satisfatórios e pode ser aplicada na determinação das relações IDF para outras regiões que não possuam pluviógrafos, mas possuam pluviômetros.

\section{REFERÊNCIAS BIBLIOGRÁFICAS}

ABUBAKARI, S.; KUSI, K. A.; XIAOHUA, D. Revision of the Rainfall Intensity Duration Frequency Curves for the City of Kumasi-Ghana. The International Journal of Engineering And Science. v.6, n.1, p.51-56, 2017.

ARAGÃO, R.; SANTANA, G. R. de; COSTA, C. E. F. F. da.; CRUZ, M. A. S.; FIGUEIREDO, E. E. de; SRINIVASAN, V. S. Chuvas intensas para o estado de Sergipe com base em dados desagregados de chuvas diárias. Revista Brasileira de Engenharia Agrícola e Ambiental, v.17, n.3, p. 243252, 2013.

BATISTA, M. L. Precipitação máxima diária anual na região Sudeste do Brasil: distribuição de probabilidade e análise espacial. 2013. 69 f. Dissertação (PósGraduação em Recursos Hídricos em Sistemas Agrícolas) - Universidade Federal de Lavras. Lavras, 2013.

BERTONI, J. C. TUCCI, C. E. M. Precipitação. In: TUCCI, C. E. M. (Org.). Hidrologia: ciência e aplicação. Porto Alegre: Ed. Universidade/UFRGS: ABRH, 2001. ed 2. p.25-33.

CAMARGO, A. P. de.; SENTELHAS, P. C.; Avaliação do desempenho de diferentes métodos de estimativa da evapotranspiração potencial no estado de São Paulo. Brasil. Revista Brasileira de Agrometeorologia, v.5, n.1, p.89-97, 1997.

CAMPOS, A. R.; SILVA, J. B. L.; SANTOS, G. G.; RATKE, R. F.; AQUINO, I. O. Estimate of intense rainfall equation parameters for rainfall stations of the Paraiba State, Brazil. Pesquisa Agropecuária Tropical, v.47, n.1, p.1521, 2017.

CARDOSO, C. O.; BERTOL, I.; SOCCOL, O. J.; SAMPAIO, C. A. P. Generation of intensity duration frequency curves and intensity temporal variability pattern of intense rainfall for Lages/SC. Brazilian Archives of Biology and Technology, v.57, n.2, p.274-283. 2014.

CASTRO, A. L. P. de; SILVA, C. N. P.; SILVEIRA, A. Curvas Intensidade-Duração-Frequência das precipitações extremas para o município de Cuiabá (MT). AmbiênciaRevista do Setor de Ciências Agrárias e Ambientais, v.7, n.2, p.305-315, 2011.
CETESB - Companhia de Tecnologia de Saneamento Ambiental. Drenagem urbana: manual de projeto. 1.ed. São Paulo: DAEE/CETESB, 1986. 466p.

CHOI, Y. H.; CHOI, J. D. Design Frequency Decision For Hydraulic Structures Due To Heavy Storm. In: Hydrologic Modeling: Proceedings of the International Conference on Water, Environment, Ecology, Socio-economics, and Health Engineering, 1999, Seoul National University, Seoul, Korea. Proceedings... Seoul, Korea: Water Resources Publication, 1999. p. 247.

COSTA, A. R. da.; PRADO, L. A. Espacialização de chuvas intensas para o estado de Goiás e sul de Tocantins. Revista Engenharia Agrícola, v.23, n.2. p.268-276, 2003.

COSTA, A. R. da.; SIQUEIRA, E. Q. de.; MENEZES FILHO, F. C. M. de. Curso básico de hidrologia urbana: nível 3. Realização Núcleo Regional Centro-Oeste de capacitação e Extensão Tecnológica em Saneamento Ambiental. Brasília: ReCESA, 2007, 130 p.

DAMÉ, R. de C. F.; TEIXEIRA, C. F. A.; TERRA, V. S. S. Comparação de diferentes metodologias para estimativa de curvas intensidade-duração-frequência para Pelotas RS. Revista Engenharia Agrícola, v.28, n.2, p.245-255, 2008.

DE PAOLA, F.; GIUGNI, M.; TOPA, M. E.; BUCCHIGNANI, E. Intensity-Duration-Frequency (IDF) rainfall curves, for data series and climate projection in African cities. Springerplus. v.3, p.1-18., 2014.

FADHEL, S.; RICO-RAMIREZ, M. A., HAN, D. Uncertainty of Intensity-Duration-Frequency (IDF) curves due to varied climate baseline periods. Journal of hydrology, v.547, p.600-612, 2017.

FARIAS, J. A. M.; SILVA, J. F. R. e.; COELHO, L. da S. Determinação de equação IDF, utilizando regressão linear em base logarítmica. In: Simpósio Brasileiro de Recursos Hídricos, 20, 2013, Bento Gonçalves. Anais... Rio Grande do Sul: ABRH, 2013. p.2.

GARCIA, S. S.; AMORIM, R. S. S.; COUTO, E. G.; STOPA, W. $H$. Determinação da equação intensidade-duraçãofrequência para três estações meteorológicas de Estado de Mato Grosso. Revista Brasileira de Engenharia Agrícola Ambiental, v.15, n.6, p. 575-581, 2011.

GHOSH, S.; MISTRI, B. Performance of D.V.C. in Flood Moderation of Lower Damodar River, India and Emergent Risk of Flood. Eastern Geographer. v.19, n.1, p.55-66, 2013.

IBGE - INSTITUTO BRASILEIRO DE GEOGRAFIA E ESTATÍSTICA. Cidades. Rio de Janeiro, 2016. Disponível em:

<http://www.cidades.ibge.gov.br/painel/historico.php?l ang $=\&$ codmun $=521010>$. Acesso em 28/06/2016.

LANNA, A. E. Elementos de estatística e probabilidades. 
In: TUCCI, C. E. M. (Org.). Hidrologia: ciência e aplicação. Porto Alegre: Ed. Universidade/UFRGS: ABRH, 2001. ed 2. p.79-176.

MAMOON, A. A.; JOERGENSEN, N. E.; RAHMAN, A. QASEM, $H$. Derivation of new design rainfall in Qatar using L-moment based index frequency approach. International Journal of Sustainable Built Environment, v.3, n.1, p.111-118, 2014.

MARTINEZ JÚNIOR, F. M.; MAGNI, N. L.G. Equações de chuvas intensas do estado de São Paulo. São Paulo: Escola Politécnica da Universidade de São Paulo, 1999. $125 \mathrm{p}$.

MORUZZI, R. B.; OLIVEIRA, S. C. de. Relação entre intensidade, duração frequência de chuvas em Rio Claro, SP: métodos e aplicação. Teoria e Prática na Engenharia Civil, n.13, v.9, p. 59-68, 2009.

NAGHETTINI, M.; PINTO, E. J. de. Hidrologia Estatística. Belo Horizonte: CPRM - Serviço Geológico do Brasil, 2007. 552 p.

OLIVEIRA, L. F. C. de.; ANTONINI, J. C. dos A.; FIOREZE, A. P.; SILVA, M. A. S da. Métodos de estimativa de precipitação máxima para o Estado de Goiás. Revista Brasileira de Engenharia Agrícola e Ambiental, v.12, n.6, p. $620-625,2008$.

OLIVEIRA, L. F. C. de.; CORTÊS, F. C.; WEHR, T. R.; BORGES, L. B.; SARMENTO, P. H. L.; GRIEBELER, N. P. Intensidadeduração-frequência de chuvas intensas para localidades no estado de Goiás e Distrito Federal. Pesquisa Agropecuária Tropical, v.35, n.1, p.13-18, 2005.

OLIVEIRA, L. F. C. de.; VIOLA, M. R.; PEREIRA, S.; MORAIS, N. R. de. Modelos de predição de chuvas intensas para o estado do Mato Grosso, Brasil. Revista Ambiente e Água, v.6, n.3, p.274-290, 2011.

QAMAR, M. U.; AZMAT, M.; SHAHID, M. A.; GANORA, D.; AHMAD, S.; CHEEMA, M. J. M.; FAIZ, M. A.; SARWAR, A.; SHAFEEQUE, M.; KHAN, M.I. Rainfall Extremes: a Novel Modeling Approach for Regionalization, Water Resources Management, v.31, n.6, p.1975-1994, 2017.

RODRIGUES, J. O.; ANDRADE, E. M. de; OLIVEIRA, T. S. de; LOBATO, F. A. de O. Equações de intensidade-duraçãofrequência de chuvas para localidades de Fortaleza e Pentecoste, Ceará. Scientia Agraria, v.9, n.4, p.511-519, 2008.

SHARMA, M. A.; SINGH, J. B. Use of Probability Distribution in Rainfall Analysis. New York Science Journal. v.3, n.9, p.40-49. 2010.

SHRESTHA, A.; BABEL, M. S.; WEESAKUL, S.; VOJINOVIC, Z. Developing Intensity-Duration-Frequency (IDF) Curves under Climate Change Uncertainty: The Case of Bangkok, Thailand. Water. v.9, n.2, 2017.
SILVA, S. R.; ARAÚJO, G. R. de S. Algoritmo para determinação da equação de chuvas intensas. Revista Brasileira de Geografia Física, v.6, n.5, p. 1371-1383, 2013.

SOUZA, R. O. R. de M.; SCARAMUSSA, P. H. M.; AMARAL, M. A. C. M. do; NETO, J. A. P.; PANTOJA, A. V.; SADECK, L. W. R. Equações de chuvas intensas para o estado do Pará. Revista Brasileira de Engenharia Agrícola e Ambiental, v.16, n.9, p.999-1005, 2012.

STEFFEN, J. L.; RONDON, M. A. C. Determinação da vazão de projeto em bacias urbanas. In: Congresso Interamericano de Engenharia Sanitária e Ambiental, XXVII, 2000, Porto Alegre. Anais... Rio de Janeiro: ABES, 2000.

SUBRAMANYA, K. Engineering Hydrology, 4 ed., New Delhi: McGraw-Hill Education, 2013. 534 p.

TUCCI, C. E. M. Hidrologia: ciência e aplicação. In: TUCCI, C. E. M. (Org.). Hidrologia: ciência e aplicação. Porto Alegre: Ed. Universidade/UFRGS: ABRH, 2001. 2. ed. p.2533.

VU, M. T.; RAGHAVAN, V. S.; LIONG, S.Y. Deriving shortduration rainfall IDF curves from a regional climate model. Natural Hazards. v.85, n.3, p.1877-1891. 2017.

WATANABE, F. M. Análise do método de Gumbel para cálculo de vazões de dimensionamento de vertedouros. 2013. 87 f. Monografia (Graduação em Sistemas de Energia e Automação) - Escola de Engenharia de São Carlos, Universidade de São Paulo, São Carlos, 2013.

WILLMOTT, C. J. On the validation of models. Physical Geography, v.2, p.184-194, 1981.

ZHAO, L.; XIA, J.; SOBKOWIAK, L.; WANG, Z.; GUO, F. Spatial Pattern Characterization and Multivariate Hydrological Frequency Analysis of Extreme Precipitation in the Pearl River Basin, China. Water Resources Management. v.26, n.12, p.3619-3637, 2012. 